\title{
Sur la composition chimique des laits tropicaux; influence du stade de lactation sur les teneurs en graisse, lactose, calcium et phosphore ${ }^{(*)}$
}

\author{
par Cl. LABOUCHE et A. PEYTAVIN
}

\begin{abstract}
Les premières analyses de lait effectuées en A.O.F. remontent déjà à de nombreuses années. Pluchon et Ginet (7), au Togo, ne purent alors établir une moyenne pour les teneurs en matière grasse et en caséine étant donné la dispersion des résultats obtenus. Dufour (3), au Sénégal, signalait la teneur importante en matiere grasse et en potassium des laits de mélange qu'il examinait, et leur déficit en phosphore, en chlore, en sodium et en magnésium. Les laits prélevés en saison des pluies se montraient plus riches en beurre, en lactose et en caséine que ceux recueillis en saison sèche.
\end{abstract}

Auparavant, Curasson (1), au Soudan français, avait mentionné, sans fournir de précisions supplémentaires et sans doute en raison du faible nombre d'échantillons analysés, des variations de la composition du lait en fonction du stade de lactation. Depuis ces travaux, il ne semble pas que des études systématiques aient été poursuivies afin de fixer la composition chimique du lait et par conséquent sa valeur alimentaire tant pour le veau que pour l'homme.

Dans cet ordre d'idée, nous avons cherché a préciser l'influence du stade de lactation sur la composition du lait des vaches tropicales. Nous ne mentionnerons que les premiers résultats obtenus: ceux-ci concernent la matière grasse, le lactose, le phosphore et le calcium.

\section{PROTOCOLE}

Les 12 femelles utilisées dans cette étude sont des croisements zébu $\times$ taurin (Bos indicus $\times$ Bos taurus), effectuant leur $3^{\text {e }}$ lactation. Les animaux pacagent en brousse. Le fourrage spontané consommé comprend, pour les zones sableuses de pacage, parmi les graminées Andropogon amplectens, Digit ri, chevalierii, Cenchrus biflorus, Perotis indica, et parmi les légumineuses Tephrosia platycarpa, Tephrosia linearis, Indigofera. Les dépressions humifères ne présentent pas de légumineuses et on y trouve Echinochloa colona, Echinochloa pyramidalis, Pennisetum pedicellatum et Imperata cylindrica, On donne chaque jour à chaque animal, environ un kilogramme de tourteau d'arachide (*). Aucune complémentation minérale n'est assurée. Les prélèvements de lait sont effectués hebdomadairement au cours du contrôle laitier. Un soin tout particulier est pris afin d'obtenir une vidange totale de la mamelle à chaque traite.

Les matières grasses sont déterminées par la méthode de Gerber, le lactose par celle de Lane et Eynon (5), le calcium par manganimétrie après minéralisation nitroperchlorique et précipitation de l'oxalate, le phosphore par photocolorimétrie du bleu de molybdène obtenu en milieu sulfitique et en présence d'hydroquinone après minéralisation nitroperchlorique. Les déterminations sont effectuées chaque semaine pour la matiere grasse et tous les quinze jours pour les autres composants.

Les résultats obtenus sont collationnés et les valeurs moyennes calculées pour chaque stade de lactation (semaine ou quinzaine). Dans chaque cas, une étude statistique est entreprise pour vérifier l'action éventuelle du temps sur la composition du lait, par comparaison de la variance liée au temps à la variance résiduelle. Si cette action n'est pas significative: les chiffres obtenus sont représentés par leur moyenne et l'écart type, l'erreur standard de la moyenne et les limites de fluctuations d'échan-

(*) Cette étude a fait l'objet d'une commuhication à l'occasion du

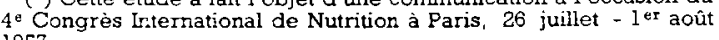
1957.

(*) Tourteau d'arachide industriel, de fabrication locale, obtenu par pression et épuisement par l'essence légère. 
tillionnage de la moyenne vraie sont déterminés. Si l'action du temps est significative, la possibilité d'une régression linéaire est testée par la signification du coefficient de pente et par comparaison de la variance liée aux déviations par rapport à la droite à la variance résiduelle. Pour chaque régression les fluctuations d'échantillonnage des paramètres sont calculées ( $\theta$ ).

Les saillies se sont effectuées librement dans notre troupeau et parfois précocement. Etant donné l'action connue de la gestation sur la production laitière et les corrélations susceptibles d'exister entre le volume du lait secrété et sa composition, nous avons divisé la lactation en deux parties : le début de lactation, d'une part, et la fin de lactation comptée à partir de la saillie. Les chiffres obtenus en début de lactation couvrent une période de 12 semaines.

\section{RÉSULTATS}

Les résultats obtenus sont consignés dans les tableaux $1,2,3,4$ et les graphiques $1,2,3$.

\section{Matière grasse.}

Au cours des premières semaines de lactation, la teneur en matière grasse du lait diminue progressivement. Cette diminution, très apparente pour le lait du soir, n'est pourtant pas statistiquement significative en raison de la dispersion des données recueillies. Les teneurs moyennes relevées pour le lait de la journée, celui du matin et celui du soir sont respectivement de $40,56 \pm 1,36 ; 37,39 \pm 1,20$; $44,76 \pm 1,80 \mathrm{~g} /$ litre. Le lait du soir est significativement plus riche que le lait du matin.

A partir de la saillie, le lait s'enrichit en matière grasse et cette augmentation peut se traduire par une régression linéaire en fonction du temps. Cet accroissement, important, avoisine 2,5 grammes par litre et par semaine. Au moment du tarissement, la teneur moyenne est comprise entre 72 et $89 \mathrm{~g} /$ litre pour le lait du matin et 80 et $98 \mathrm{~g} /$ litre pour le lait du soir. Les valeurs correspondantes au moment de la saillie sont comprises d'une part entre 38 et $42 \mathrm{~g} /$ litre et 43 à $45 \mathrm{~g} /$ /itre d'autre part. Le lait, au tarissement, est donc 2 fois plus riche que le lait prélevé au moment de la saillie.

\section{Lactose.}

Au cours des premières semaines de lactation, le temps n'agit pas significativement sur la teneur en lactose du lait. Les teneurs moyennes en gramme par litre sont respectivement de $52,37 \pm 0,51$; $53,03 \pm 0,63 ; 51,68 \pm 0,45$ pour le lait de la journée, le lait du matin et celui du soir. Ces teneurs ne sont pas significativement différentes les unes des autres.
Au moment de la saillie les teneurs moyennes sont de $53,84 \pm 0,76 ; 53,44 \pm 0,76 ; 54,03 \pm 0,831$ pour le lait de la journée, celui du matin et celui du soir.

Après la saillie, le lait s'appauvrit en lactose. La pente de la régression est pratiquement la même pour le lait du matin, du soir ou de la journée et elle est proche de 1 gramme par litre et par quinzaine.

\section{Calcium.}

L'action du temps ne se fait sentir ni en début ni en fin de lactation. Dans les deux cas, les teneurs en calcium du lait du matin, du soir et de la journée ne sont pas significativement différentes. En début de lactation, les chiffres moyens obtenus sont de $1,65 \mathrm{~g} /$ litre à $0,2 \mathrm{~g}$ près et en fin de lactation de $1,75 \mathrm{~g} /$ litre à $0,07 \mathrm{~g}$ près.

\section{Phosphore.}

La teneur en phosphore paraît insensible à l'intervention du temps tant au début qu'en fin de lactation et les valeurs obtenues ne sont pas significativement différentes les unes des autres pour le lait de la journée, le lait du matin et celui du soir. Les moyennes relevées sont en début de lactation de 1,2 g/litre et en fin de lactation de 1,15 g/litre.

\section{Rapport phospho-calcique .}

Le rapport phospho-calcique est presque constant étant donné la fixité des teneurs en $\mathrm{P}$ et $\mathrm{Ca}$. II s'élève à environ 1,5.

\section{DISCUSSION DES RÉSULTATS}

\section{Matière grasse.}

Si on se reporte aux courbes moyennes de lactation observées chez nos animaux, courbes de type parabolique (6), comparables à celles obtenues. dans la métropole par Delage, Leroy et Poly (2), on remarque que la production au cours des 12 premières semaines de lactation couvre environ 40 p. 100 de la production laitière totale. Ainsi pendant cette période, quantitativement importante, la teneur en matière grasse, de l'ordre de $40 \mathrm{~g} /$ litre, s'écarte moins de la teneur moyenne des laits métropolitains (38 g/litre) que de la teneur moyenne généralement adoptée pour les laits tropicaux (50 g/litre). Cette dernière donnée demande donc à être nuancée.

En milieu tempéré, une diminution de la teneur en matière grasse au cours des premières semaines de la lactation a été signalée et, dans les graphiques publiés par Hanson et coll. (1949) (4), un minimum se situe à la 8 e' semaine de lactation. Nous avons observé une diminution' comparable, mais non significative. Ceci est explicable si l'on admet une 
GRAPHIQUE 1 .. COMPOSITIOH DU LAIT DU MATIN EN FONCTION DU STADE DE LACTATION

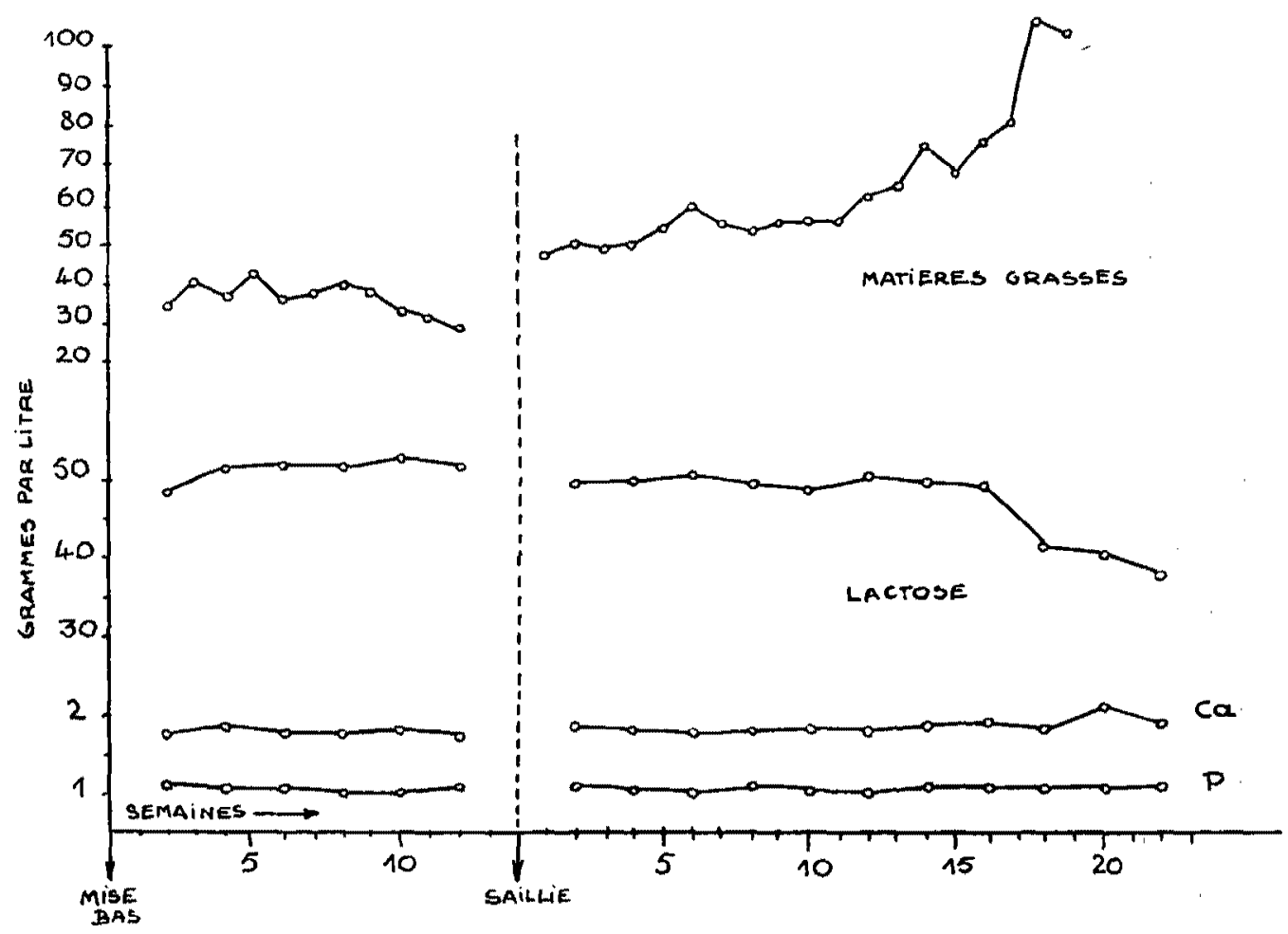

gRAPHIQUE 2.. COMPOSITION DU LAIT DU SOIR EN FONCTION DU STADE DE LACTATION

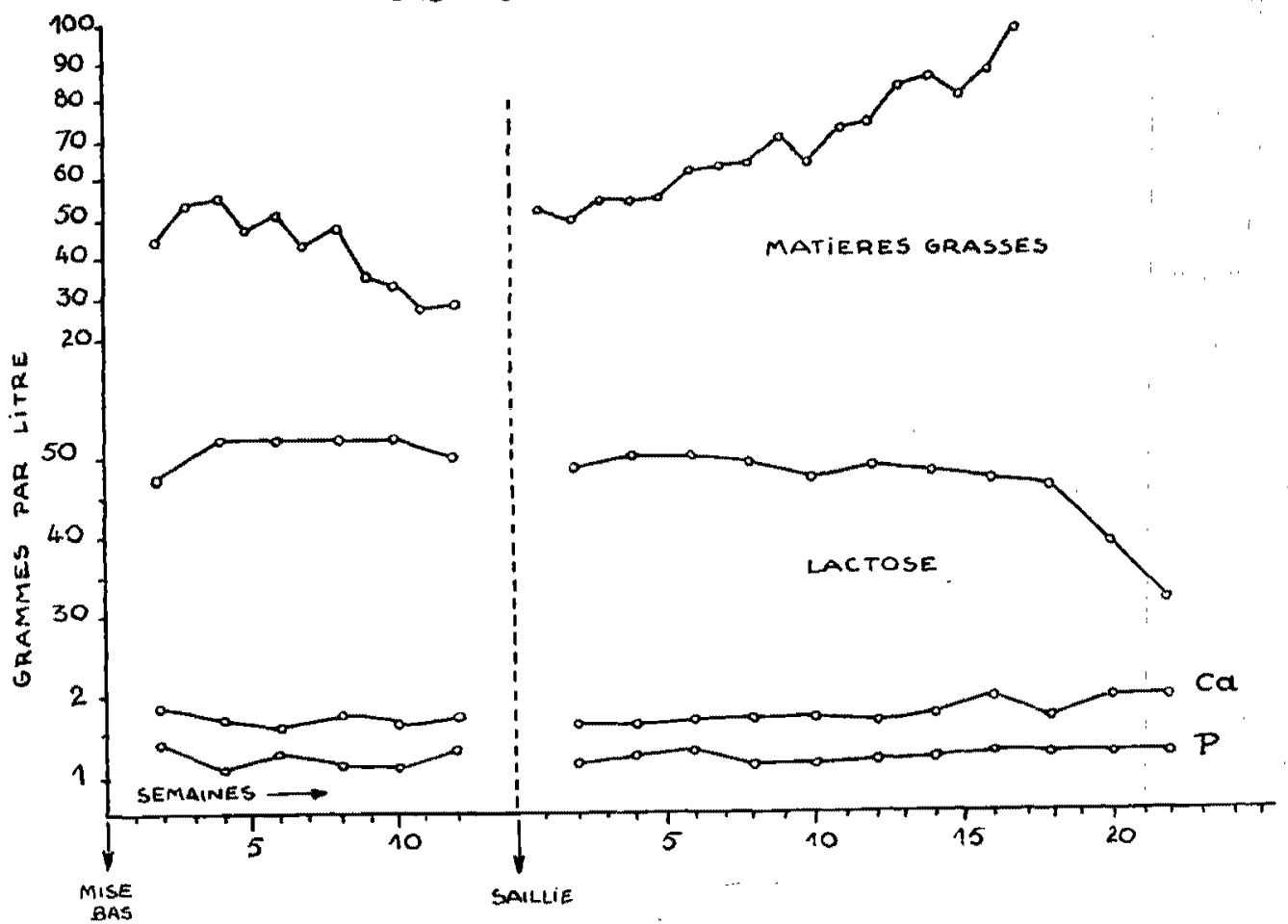


GRAPHIQUE 3.. COMPOSITION DU LAIT DE LA JOURMEE EM FONCTION DU STADE DE LACTATION

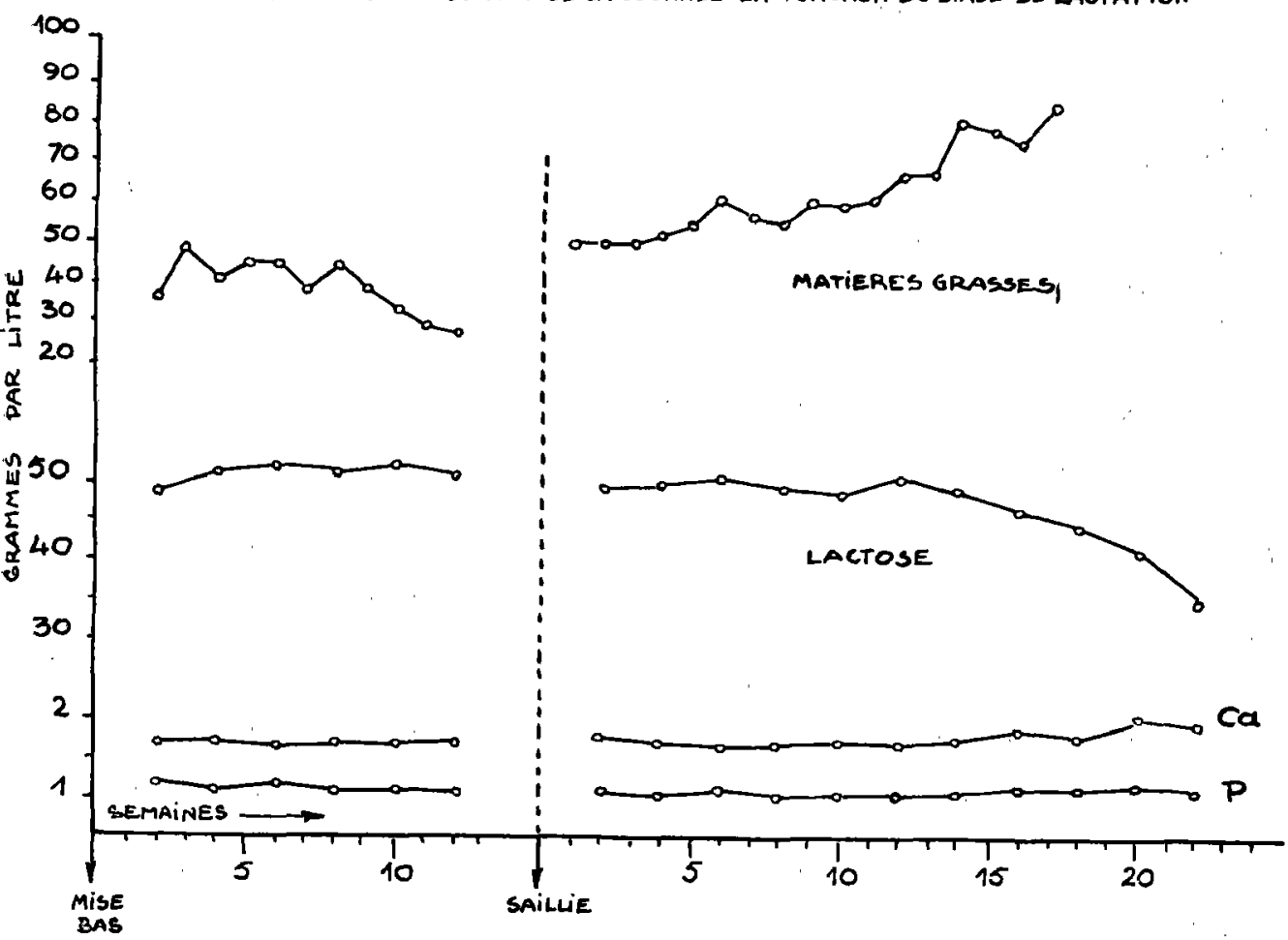

corrélation négative entre la teneur en matière grasse et la quantité de lait, car dans nos courbes de lactation nous n'avons pas observé d'augmentation significative de la production laitière au cours des premières semaines de lactation (6). Signalons d'autre part que cette diminution de la teneur en matière grasse n'a pas été signalée en Inde (8).

Après la saillie, la teneur en matière grasse augmente. Cette augmentation est précoce, surtout pour le lait du soir, et survient même avant que la production laitière ait sensiblement diminué. Elle rappelle les phénomènes comparables observés en milieu tempéré (4) ainsi qu'en Inde par Kothavalla et Kartha en 1939 (8). Cependant, l'augmentation signalée par ces auteurs se produit tout au long de la lactation et paraît moins intense que celle que nous observons, puisque le taux d'augmentation par mois ne serait que de 0,06 à 0,07 p. 100 .

Les teneurs relevées au moment du tarissement sont très élevées mais on aura garde de les comparer aux valeurs correspondantes métropolitaines, car les productions au moment du tarissement chez la vache tropicale sont toujours très faibles et, dans nos observations, sont souvent inférieures à $250 \mathrm{ml}$ de lait de traite.

\section{Lactose.}

Au cours des premières semaines de lactation, la teneur en lactose est comparable à celle donnée pour les laits de pays tempérés : 48 p. 1000 (Johansson et Claesson 1957) (4); 47,5 à 55 p. 1.000 (Vivario, 1953) (10); d'autre part, Sen (8) donne pour les laits analysés en Inde une teneur de 50,6 p. 1.000 et Curasson (1), au Soudan français, le chiffre de $47,5 \mathrm{~g} /$ litre. Par contre nos valeurs sont inférieures à celles de Pigneur (1) et supérieures à celles de Els (1), au Congo belge : $100 \mathrm{~g} /$ /itre dans le premier cas et $38 \mathrm{~g} /$ litre dans le deuxième. Par contre elles confirment les chiffres donnés pour la presqu'île du Cap-Vert par Dufour (3).

Après la saillie nous assistons à une diminution régulière du lactose, diminution de $1 \mathrm{~g} /$ litre et par quinzaine. Cette chute paraît plus rapide que celle remarquée en milieu tempéré sur les graphiques de Hansson et coll. (1949) (4) et nous n'avons pas observé, comme ces auteurs, d'augmentation du lactose au moment du tarissement. Signalons que Vivario (1953) (10) a noté une diminution continuelle de la teneur en lactose qui conduit au tarissement, à des valeurs très basses de $15 \mathrm{~g} /$ litre. 
TABLEAT I

TENEUR EN MATIERE GRASSE, EXPRINEE EN GRAMTES PAR LITRE DE LAIT, ET EU FOHCTIOH DU STADE DE LACTATION. DEBUT DE IACTATION

\begin{tabular}{|c|c|c|c|}
\hline Semaines de lactation & Layt du matin & Lait du soir & Lait de la journée \\
\hline $\begin{array}{c}2 \\
3 \\
4 \\
5 \\
6 \\
7 \\
8 \\
9 \\
10 \\
11 \\
12 \\
\text { Moyenne } \\
\text { Ecart type } \\
\text { Limite supérieure de la moyenne vraie } \\
\text { Limite inférieure de la moyenne vraie } \\
\text { Action du temps }\end{array}$ & $\begin{array}{c}34,93 \\
40,06 \\
37,75 \\
42,57 \\
36,14 \\
38,25 \\
40,33 \\
39,33 \\
33,75 \\
31,50 \\
29,75 \\
37,39 \\
9,88 \\
1,198 \\
39,74 \\
35,04 \\
\text { non significative }\end{array}$ & $\begin{array}{c}43,78 \\
53,25 \\
54,14 \\
48,00 \\
51,66 \\
42,81 \\
48,41 \\
36,10 \\
33,37 \\
28,50 \\
29,85 \\
44,76 \\
14,48 \\
1,795 \\
48,28 \\
41,24 \\
\text { non significative }\end{array}$ & $\begin{array}{c}36,28 \\
48,93 \\
40,28 \\
45,78 \\
45,71 \\
39,43 \\
44,17 \\
39,64 \\
33,87 \\
29,83 \\
29,62 \\
40,56 \\
11,05 \\
1,360 \\
43,22 \\
37,90 \\
\text { non significative }\end{array}$ \\
\hline
\end{tabular}

FIN DE LACTATION

\begin{tabular}{|c|c|c|c|}
\hline Semaines depuis la saillie & Lait du matin & Lait du soir & Lait de la journée \\
\hline $\begin{array}{c}1 \\
2 \\
3 \\
4 \\
5 \\
6 \\
7 \\
8 \\
9 \\
10 \\
11 \\
12 \\
13 \\
14 \\
15 \\
16 \\
17 \\
18 \\
19 \\
\text { Action du tenps } \\
\text { Test de linéarité } \\
\text { a } \\
\text { b }\end{array}$ & $\begin{array}{c}48,58 \\
50,60 \\
49,40 \\
50,25 \\
55,30 \\
60,50 \\
55,75 \\
52,80 \\
55,60 \\
56,40 \\
56,70 \\
63,70 \\
64,90 \\
73,50 \\
66,67 \\
73,00 \\
80,33 \\
109,50 \\
105,33 \\
+ \\
+ \\
40,61 \pm 2,03 \\
2,37 \pm 0,38\end{array}$ & $\begin{array}{c}51,42 \\
50,00 \\
53,00 \\
53,20 \\
56,40 \\
62,20 \\
62,80 \\
64,20 \\
70,20 \\
63,10 \\
71,80 \\
72,60 \\
83, \infty 0 \\
84,75 \\
80,00 \\
87,00 \\
98,16 \\
\end{array}$ & $\begin{array}{c}50,10 \\
50,30 \\
50,40 \\
52,30 \\
55,80 \\
61,90 \\
57,50 \\
56,50 \\
60,50 \\
59,80 \\
61,40 \\
66,60 \\
66,40 \\
80,50 \\
78,70 \\
75,50 \\
84,60 \\
\vdots \\
+ \\
+ \\
21,23 \pm 1,90 \\
2,49 \pm 0,41\end{array}$ \\
\hline
\end{tabular}


TENEUR EN IACTOSE HYDRATE, EN GRAMUES PAR IITRE DE LAIT ET EN FONCTION DU STADE DE LACTATION DEBUT DE LACTATION

\begin{tabular}{|c|c|c|c|}
\hline Quinzaines depuis la mise-bas & Lait dụ matin & Lait du soir & Lait de la journée \\
\hline 1 & 49,34 & 47,54 & 48,66 \\
3 & 52,43 & 52,67 & 52,55 \\
4 & 53,38 & 52,58 & 52,98 \\
5 & 52,81 & 52,46 & 52,68 \\
6 & 55,56 & 52,67 & 53,99 \\
Moyenne & 53,68 & 50,85 & 52,29 \\
Ecart type & 53,028 & 51,677 & 52,372 \\
Erreur standard de la muyenne & 3,91 & 2,78 & 3,18 \\
Limite supérieure de la moyenne vraie & 0,626 & 0,45 & 0,51 \\
Limite inférieure de la moyeme vraie & 54,255 & 52,531 & 53,370 \\
Action du temps & 51,801 & 50,803 & 51,374 \\
\hline
\end{tabular}

IIN DE LACTATION

\begin{tabular}{|c|c|c|c|}
\hline Quinzaines depuis la saillie & Lait du matin & Lait du soir & Lait de Ia journée \\
\hline $\begin{array}{c}1 \\
2 \\
3 \\
4 \\
5 \\
6 \\
7 \\
8 \\
9 \\
10 \\
11 \\
\text { Action du temps } \\
\text { Test de linéarité } \\
a \\
\text { b }\end{array}$ & $\begin{array}{c}49,17 \\
50,17 \\
51,42 \\
49,77 \\
48,20 \\
51,14 \\
49,81 \\
48,60 \\
43,50 \\
42,05 \\
38,14 \\
\text { significative } \\
+ \\
53,445 \pm 0,762 \\
-0,942 \pm 0,257\end{array}$ & $\begin{array}{c}49,77 \\
50,10 \\
50,42 \\
49,64 \\
48,30 \\
49,39 \\
49,00 \\
47,49 \\
46,80 \\
38,92 \\
33,12 \\
\text { significative } \\
+ \\
54,026 \pm 0,831 \\
-1,168 \pm 0,283\end{array}$ & $\begin{array}{c}49,23 \\
50,13 \\
50,82 \\
49,66 \\
48,28 \\
51,08 \\
49,74 \\
47,52 \\
45,15 \\
41,96 \\
35,68 \\
\text { significative } \\
+ \\
53,844 \pm 0,764 \\
-1,004 \pm 0,272\end{array}$ \\
\hline
\end{tabular}


TENEUR EN CAICIUM, EXPRINEE EN GRAMTES PAR LITRE LE LAIT, ET EN FONCTION DU STADE DE LACTATION DEBU'T DE LACTATION

\begin{tabular}{|c|c|c|c|}
\hline Quinzaines depuis la mise-bas & Lait du natin & Lait du soir & Lait de la journée \\
\hline 1 & 1,63 & 1,80 & 1,70 \\
2 & 1,75 & 1,67 & 1,69 \\
3 & 1,59 & 1,60 & 1,59 \\
4 & 1,64 & 1,71 & 1,66 \\
5 & 1,70 & 1,63 & 1,66 \\
6 & 1,61 & 1,72 & 1,70 \\
Moyenne & 1,663 & 1,681 & 1,658 \\
Ecart type & 0,169 & 0,187 & 0,380 \\
Eimite supérieure de la moyenne vraie & 0,029 & 0,034 & 0,067 \\
Limite inférieure de la moyenne vraie & 1,720 & 1,748 & 1,792 \\
Action du temps & 1,606 & 1,614 & 1,524 \\
\hline
\end{tabular}

FIN DE IACTATION

\begin{tabular}{|c|c|c|c|}
\hline Quinzaines depuis Ia saillie & Iait du matin & Lait du soir & Lait de la journée \\
\hline $\begin{array}{c}1 \\
2 \\
3 \\
4 \\
5 \\
6 \\
7 \\
8 \\
9 \\
10 \\
11 \\
\text { Moyenne } \\
\text { Ecart type } \\
\text { Limite supérieure de la moyenne vraie } \\
\text { Limite inférieure de la moyeme vraie } \\
\text { Action du temps }\end{array}$ & $\begin{array}{c}1,85 \\
1,72 \\
1,57 \\
1,71 \\
1,67 \\
1,65 \\
1,84 \\
1,94 \\
1,87 \\
2,21 \\
1,86 \\
1,812 \\
0,310 \\
0,042 \\
1,854 \\
1,730 \\
\text { non significative }\end{array}$ & $\begin{array}{c}1,69 \\
1,65 \\
1,71 \\
1,71 \\
1,71 \\
1,67 \\
1,77 \\
2,03 \\
1,77 \\
2,00 \\
2,04 \\
1,779 \\
0,212 \\
0,034 \\
1,84 \\
1,720 \\
\text { non signicative }\end{array}$ & $\begin{array}{c}1,78 \\
1,67 \\
1,62 \\
1,71 \\
1,69 \\
1,66 \\
1,82 \\
1,98 \\
1,80 \\
2,10 \\
1,98 \\
1,800 \\
0,509 \\
0,069 \\
1,87 \\
1,730 \\
\text { non significative }\end{array}$ \\
\hline
\end{tabular}


TENEUR EN PHOSPHORE EN FONCTION DU STADE DE LACTATION ET EXPRIMEE EN GRANDES PAR LITRE DE IAIT DEBUT DE LACTATION

\begin{tabular}{|c|c|c|c|}
\hline Quinzaines depuis la mise-bas & Lait du matin & Lait du soir & Lait de la journée \\
\hline 1 & 1,171 & 1,307 & 1,222 \\
2 & 1,125 & 1,117 & 1,121 \\
3 & 1,143 & 1,192 & 1,166 \\
4 & 1,090 & 1,080 & 1,086 \\
5 & 1,084 & 1,060 & 1,077 \\
6 & 1,182 & 1,147 & 1,143 \\
Moyenne & 1,127 & 1,135 & 1,090 \\
Ecart type & 0,106 & 0,131 & 0,090 \\
Eimite supérieure de la moyenne vraie & 0,017 & 0,022 & 1 \\
Inmite inférieure de la moyenne vraie & 1,144 & 1,178 & 0,015 \\
Action du temps & 1,110 & 1,092 & 1,156 \\
\hline
\end{tabular}

FIN DE LACTATION

\begin{tabular}{|c|c|c|c|}
\hline Quinzaines depuis la saillie & Lait du matin & Lait du soir & Lait de la journée \\
\hline $\begin{array}{c}1 \\
2 \\
3 \\
4 \\
5 \\
6 \\
7 \\
8 \\
9 \\
10 \\
11 \\
\text { Mioyenne } \\
\text { Ecart type } \\
\text { Limite supérieure de la moyenne vraie } \\
\text { Ermite inférieure de la moyenne vraie } \\
\text { Action du temps }\end{array}$ & $\begin{array}{c}1,180 \\
1,157 \\
1,075 \\
1,179 \\
1,152 \\
1,108 \\
1,187 \\
1,171 \\
1,163 \\
1,183 \\
1,190 \\
1,160 \\
0,244 \\
0,031 \\
1,221 \\
1,099 \\
\text { non significative }\end{array}$ & $\begin{array}{c}1,116 \\
1,155 \\
1,274 \\
1,096 \\
1,074 \\
1,091 \\
1,083 \\
1,204 \\
1,184 \\
1,185 \\
1,143 \\
1,142 \\
0,179 \\
0,023 \\
1,188 \\
1,096 \\
\text { non significative }\end{array}$ & $\begin{array}{c}1,143 \\
1,155 \\
1,180 \\
1,104 \\
1,136 \\
1,102 \\
1,141 \\
1,175 \\
1,170 \\
1,183 \\
1,160 \\
1,150 \\
0,144 \\
0,019 \\
1,188 \\
1,112 \\
\text { non significative }\end{array}$ \\
\hline
\end{tabular}




\section{Calcium.}

Les valeurs que nous avons recueillies sont du même ordre de grandeur que celles données pour les laits tropicaux par Dufour, à Dakar (3) : 1,4 g/litre, et Anatakrishnan (1943) : 1,27 g/litre en Inde (8) et celles avancées pour les laits de milieu tempéré par Johansson et Claesson (1957) (4): 1,2 g/litre, et par Vivario (1953) (10) : 1,2 à 1,7 g/litre. Il apparaît donc difficile de parler d'un déficit en calcium ainsi que l'avait signalé Dufour (3).

Par contre, la diminution de la teneur en calcium au moment du maximum de lactation signalé en Inde par Desai et Mathur (8), n'a pas été retrouvée, la teneur en calcium, dans nos observations, paraissant insensible à l'action du temps.

\section{Phosphore .}

La teneur en phosphore est constante et égale à $1,1 \mathrm{~g} / \mathrm{litre}$. Cette valeur est comparable à celle donnée en milieu tempéré par Johansson et Claesson (4): I g/litre, et en Inde par Anantakrishnan (1943): $0,92 \mathrm{~g} /$ litre (8). Elle est sensiblement supérieure à celle relevée par Dufour à Dakar (1937) (3): 0,7 g/litre. Nos données ne confirment donc pas le déficit en phosphore signalé par cet auteur.

En milieu tempéré, Vivario (1953) (10) donne des teneurs en $P$ beaucoup plus élevées: 2,5 g/litre, qui diminuent pour atteindre en fin de lactation, $1,6 \mathrm{~g} / \mathrm{litre}$. Ces observations, ne portant que sur un animal, ne sauraient cependant avoir une valeur générale.

\section{Rapport phospho-calcique.}

Ce rapport est sensiblement égal à celui observé en milieu tempéré ou en Inde. Il est par contre inférieur à celui que l'on peut calculer à partir des données de Dufour (3) : $\mathrm{Ca} / \mathrm{P}=2$. Cette divergence provient de la différence entre les teneurs en phosphore.

\section{CONCLUSIONS.}

La teneur en matière grasse, en lactose, en calcium et en phosphore du lait de vaches métis zébu $x$ taurin a été déterminée au cours des 12 premières semaines de lactation et de la saillie au tarissement.

$1^{0}$ Au cours des 12 premières semaines de lactation, le lait contient $40 \mathrm{~g} /$ litre de matière grasse. 40 p. 100 du lait obtenu au cours d'une lactation complète possèdent donc une teneur en matière grasse comparable à celle des laits de milieu tempéré, et nettement inférieure à la donnée classique de $50 \mathrm{~g} /$ litre que l'on affecte habituellement aux laits tropicaux.
Après la saillie, le taux de matières grasses augmente progressivement d'environ $2,5 \mathrm{~g} /$ /itre et par semaine pour atteindre au moment du tarissement des valeurs de l'ordre de 80 à $90 \mathrm{~g} / \mathrm{litre}$. Le taux de graisse au moment de la saillie est de 40 à 42 g/litre.

$2^{\circ}$ Au début de la lactation, la teneur en lactose, de l'ordre de $52 \mathrm{~g}$ /litre est pratiquement constante. Elle s'apparente aux données connues pour les laits de milieu tempéré, pour les laits de l'Inde et d'A.O.F., mais s'écarte sensiblement de celles obtenues au Congo belge.

Après la saillie, le taux de lactose diminue de 1 g/litre,quinzaine. Cette chute est plus rapide que celle observée en milieu tempéré. Au tarissement, le lactose atteint environ $35 \mathrm{~g} /$ litre, valeur inférieure à celle des milieux tempérés.

$3^{\circ}$ La teneur en calcium, pratiquement constante au cours de la lactation, avoisine 1,70 g/litre. Elle est du même ordre de grandeur que la teneur en calcium des laits de pays tempérés, tout en lui demeurant légèrement supérieure. Il n'existe pas de déficit calcique.

40 La teneur en phosphore, $1,1 \mathrm{~g} / \mathrm{litre}$, est constante. Identique aux données des milieux tempérés, elle est par contre supérieure aux estimations antérieurement acquises au Sénégal. Il n'existe pas de déficit en phosphore.

Le rapport $\mathrm{Ca} / \mathrm{P}$, normal, est égal à 1 ,5. Il 'est inférieur à celui déjà énoncé pour le Sénégal.

En résumé, du point de vue de la graisse, du lactose, du calcium et du phosphore, le lait de vaches tropicales est pratiquement identique aux laits des milieux tempérés, pendant les 12 premières semaines de lactation au cours desquelles 40 p. 100 du lait produit dans une lactation complète sont secrétés. Après la saillie, les différences ne s'accusent que pour la matière grasse et le lactose. L'augmentation de la matière grasse est plus accusée qu'en milieu tempéré tandis que le lactose diminue plus rapidement que pour les laits métropolitains.

Laboratoire Fédéral de l'Elevage « Georges Curasson » à Dakar.

Directeur: P. Mornet.

\section{BIBLIOGRAPHIE:}

1. CURASSON (G.). - Note sur la composition du lait des vaches africaines et son utilisation dans l'alimentation des enfants et des adultes. Bull. Soc. Path. Exo;, (1933), 26, 536 . 
2. DELAGE (J.) LEROY (A.-M.), POLY (J.). Une étude sur les courbes de lactation. Ann. Zoot., (1953), 3, 225-267.

3. DUFOUR (V.). - Etude sur les laits consommés à Dakar. Ann. Méd. Pharm. Col., (1937), 25, $\mathrm{n}^{0} 1,87$.

4. JOHANSSON (I.), CLAESSON (O.). - Factors affecting the composition of milk. In Hammond (J.). Progress in the physiology of farm animals, vol. 3, London, 1957, Butterwoths, 1 vol.

5. JOURNAL OFFICIEL DE LA REPUBLIQUE FRANÇAISE. - Analyse physique et chimique du lait (méthodes officielles). Paris, 1954.

6. LABOUCHE (C.). - Physiologie de la lactation en milieu tropical. I. Etude des courbes de lactation recueillies en zone sub-gui- néenne de la presqu'île du Cap-Vert (Séné-

gal). Rev. Elev. Med. Vét. Pays Trop. (1957), 10, no 1, 27-39.

7. PLUCHON (J.-P.), GINET (H.-M.), -- Etude sur le lait consommé à Lomé (Togo). Ann. Méd. Pharm. Col. (1932), 30, 493-508.

8. SEN (K.-C.). - Animal nutrition research in India. London, 1953, Mc Millan, l vol., $370 \mathrm{p}$.

9. VESSEREAU (A.). - Méthodes statistiques en biologie et en agronomie. Paris, 1948, Baillière, 1 vol. $381 \mathrm{p}$.

10. VIVARIO (R.), - Le lait. Les fluctuations naturelles de sa composition. Son contrôle analytique. In Mises au point de Chimie analytique pure et appliquée et d'analyse bromatologique, $1^{\text {re }}$ série, Paris, 1953, Masson 1 vol. $171 \mathrm{p}$.

\section{SUMMARY}

Variation in the chemical contents of milk in tropical countries. The influence of various stages of lactation on fat, lactose, calcium and phosphorus contents.

The fat, lactose, calcium and phosphorus contents of milk from crossbred cows (zebus $x$ humpless cattle) were determined during the first 12 weeks of lactation and from the mating period to drying off. As far as the above substances were concerned, milk collected from tropical countries was virtually the same as that from temperate countries during the first 12 weeks of lactation when $40 \%$ of the quantity produced during the whole period was collected (Fat $40 \mathrm{~g}$., lactose $52 \mathrm{~g}$., calcium 1,7 g., phosphorus 1,1 g. per litre). After mating the only differences observed in the composition of milk compared with that obtained during the 12 weeks after calving were in the fat and lactose contents : the fat content increased each week by 2,5 g./litre and eventually reached 80-90 g./litre at the drying off whereas the lactose content decreased each week by $0,5 \mathrm{~g} . /$ litre until reaching $35 \mathrm{~g}$ //litre.

\section{RESUMEN}

Sobre la composición quimica de las leches tropicales. Influencia del estado de lactación sobre los tenores de grasa, lactosa, calcio y fósforo.

El tenor en materia grasa, lactosa, calcio y fósforo de la leche de vacas mestizas zébu $\mathrm{x}$ taurin ha sido determinado durante las doce primeras semanas de la lactación asi como desde la cubricion hasta su finalización. Para los elementos considerados, la leche de vacas tropicales es practicamente idéntica a las leches producidas en lugares templados en el curso de las doce primeras semanas de la lactación durante las cuales es secretado el 40 p. 100 del total de la leche producida en un periodo completo de lactación (materia grasa : 40 gramos/litro; lactosa : 52 gramos/litro; calcio : 1,7 gramo/litro; fosforo : 1,1 gramo/litro).

Después de la cubrición la diferencia no se manifesta sino por cambios en la cantidad de materia grasa y lactosa (la materia grasa aumenta 2,5 gramos/litro por semana hasta llegar a 80 a 90 gramos/litro al fín del periodo; la lactosa disminuye 0,5 gramo/litro por semana hasta llegar a 35 gramos/litro al fín de la lactación). 